\begin{tabular}{|c|l|}
\hline Title & Focusing of large wave vector phonons in GaA s \\
\hline Author(s) & Tamura, Shin-ichiro; Harada, Toshinaru \\
\hline Citation & $\begin{array}{l}\text { Physical Review B, 32(8), 5245-5250 } \\
\text { https://doi.org/10.1103/PhysRevB.32.5245 }\end{array}$ \\
\hline Issue Date & 1985-10-15 \\
\hline Doc URL & http://hdl.handle.net/2115/5951 \\
\hline Rights & Copyright $\odot$ 1985 A merican Physical Society \\
\hline Type & article \\
\hline File Information & PRB32-8.pdf \\
\hline
\end{tabular}

Instructions for use 


\title{
Focusing of large-wave-vector phonons in GaAs
}

\author{
Shin-ichiro Tamura \\ Department of Engineering Science, Hokkaido University, Sapporo 060, Japan \\ Toshiharu Harada \\ Department of Applied Physics, Hokkaido University, Sapporo 060, Japan
}

(Received 1 April 1985)

\begin{abstract}
Focusing of 0- to 1.5-THz transverse-acoustic phonons in GaAs is studied on the basis of an extended rigid-ion model, which reproduces very accurately the observed dispersion curves of acoustic phonons. The shifts of phonon caustics due to lattice dispersion become conspicuous at frequencies above $1 \mathrm{THz}$. Calculated focusing patterns of ballistic phonons are found to be in good agreement with the characteristic features of phonon images obtained recently by Wolfe and Northrop. We also compare the dispersive shifts of caustics in $\mathrm{GaAs}$ with those in $\mathrm{Ge}$ and discuss its implication to the phonon-focusing experiments with large-wave-vector phonons.
\end{abstract}

\section{INTRODUCTION}

The propagation of large-wave-vector phonons photoexcited in GaAs has been a subject of great interest in recent years. ${ }^{1}$ This interest is motivated to a considerable extent by the observation by Ulbrich, Narayanamurti, and Chin of energy transport via TA (transverse-acoustic) phonons. ${ }^{2}$ However, there are some controversies whether the energy transport in high-purity GaAs occurs via long-lived dispersive TA phonons or via quasidiffusion.,3 At present there seems to be no conclusive results. The most direct experimental method which discriminates the ballistic transport from the diffusive transport is evidently to observe the focusing patterns of phonons impinging on the detector. This attempt has recently been carried out by Wolfe and Northrop. ${ }^{4}$ By a phonon-imaging method, they have observed phonon-focusing patterns in GaAs. It is found that the phonon image of a $0.25-\mathrm{mm}$-thick sample is substantially different from the longer-path (of 1.9 $\mathrm{mm}$ ) image, suggesting the ballistic transport of dispersive phonons (of about $1 \mathrm{THz}$ ) over submillimeter distances being consistent with the phonon mean-free path limited by isotope scattering. ${ }^{5}$ However, there exists no detailed analysis on the maximum frequency of phonons which constitute the ballistic component of the detected flux. The aim of this work is to predict quantitatively the dispersive effects on the TA-phonon focusing in GaAs and compare the results with experimental phonon images. We also compare the dispersive shifts of phonon caustics in GaAs with those in Ge. As a consequence it is concluded that GaAs is less dispersive than $\mathrm{Ge}$ as far as phonon focusing is concerned.

\section{LATTICE MODEL}

As in the case of Ge the dispersion relation of the TA branches in GaAs is highly nonlinear, being characterized by marked flattenings of the dispersion curves especially in the $\langle 111\rangle$ direction. [The lowest zone-boundary frequency of the TA phonons is $1.87 \mathrm{THz}$ at point $L$ (Ref.
6), which is slightly lower than $1.90 \mathrm{THz}$ in Ge (Ref. 7) at the same point.] These flattenings of the TA branches are attributed to long-range interatomic forces which may arise from electrostatic quadrupole interactions ${ }^{8}$ and ionshell forces. ${ }^{9}$ In order to discuss the focusing properties of the phonons in partially ionic crystals, we need to diagonalize the dynamical matrix of the lattice for a large number of wave vectors (of more than $10^{5}$ ) after the calculation of matrix elements consisting of the short-range forces as well as long-range Coulombic forces. Within the framework of the dipole and shell models we further require several times of matrix inversions to obtain the phonon frequencies. To save computation time we employ the rigid-ion model which does not necessitate the additional matrix inversions. However, we extend this model by adding short-range forces up to fifth nearest neighbors to ensure good fitting of the theoretical dispersion curves to experimental frequencies. The 23 parameters in total (22 parameters describing force constants and one describing the static ionic charge) are then fitted to the experimental frequencies ${ }^{6}$ and elastic constants. ${ }^{10}$

$\mathrm{GaAs}$ is a piezoelectric material and, therefore, stiffening of effective elastic constants can be expected. In the

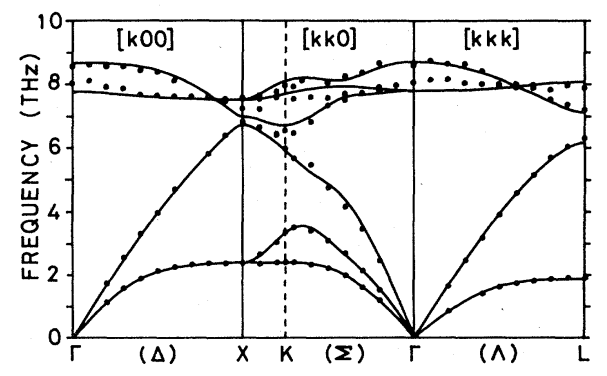

FIG. 1. Calculated phonon-dispersion curves of GaAs. Experimental frequencies are from Ref. 6. 
low-frequency limit the piezoelectric effects are estimated by the velocity change

$$
\frac{\Delta c}{c}=\frac{e_{p}^{2}}{2 \epsilon_{0} C_{44}}=1.9 \times 10^{-3},
$$

where $c$ is an appropriate phase velocity, $e_{p}$ is the piezoelectric constant, ${ }^{11} \epsilon_{0}$ is the static dielectric constant, ${ }^{12}$ and $C_{44}$ is a component of the compliance tensor. $^{10}$ Because the right-hand side of Eq. (1) is much smaller than unity, the piezoelectric effects can be safely neglected in the discussion of the focusing properties of the low-frequency phonons. In the rigid-ion model, however, the static dielectric constant is fixed at a value close to unity. Hence, the piezoelectric effects are enhanced by a factor of the order of 10 if the correct value of piezoelectric constant is included in the fitting procedure because $\epsilon_{0}=13$ for GaAs. ${ }^{12}$ Therefore, we have fitted the parameters so that the piezoelectric constant may be underestimated.

The comparison of the theory with experimental frequency data is shown in Fig. 1. Our model reproduces the frequencies of the acoustic branches relevant to the present discussion quite well, although there exist some deviations for the frequencies of the optical branches. The predicted elastic constants are $C_{11}=12.18(12.26)$, $C_{12}=5.74(5.71)$, and $C_{44}=5.95(6.00)$ in units of $10^{11}$ dyn $\mathrm{cm}^{-2}$, which should be compared with the experi- mental values (extrapolated to $0 \mathrm{~K}$ ) in the parentheses. ${ }^{10}$ More details of the results derived from this model, such as the one-phonon and two-phonon densities of states will be given elsewhere ${ }^{13}$ in relation to the study of anharmonic decay of the TA phonons in GaAs.

\section{CONSTANT-FREQUENCY SURFACES}

The focusing of phonons results from the fact that the constant-frequency surface ( $\omega$ surface) of each phonon mode spanned in the wave-vector space ( $k$ space) is distorted from a spherical shape due to the crystal anisotropy. ${ }^{14}$ The direction of the energy flow of the phonons is determined by the outward normal of the $\omega$ surface or group-velocity vector. Hence, the phonons accumulate, in general, along some directions as compared to other directions in the real space, even if the phonons are distributed uniformly in the $\mathbf{k}$ space. In Figs. 2(a)-2(d) we have plotted the $\omega$ surfaces of the TA phonons in GaAs at 0 and $1.5 \mathrm{THz}$ frequencies. (We refer to the lower and upper TA branches as T1 and T2 branches, respectively.) Even at $0 \mathrm{THz}$ these surfaces are highly nonspherical and consist generally of the concave and convex regions with positive Gaussian curvature and the saddle regions with negative Gaussian curvature, which are separated from each other by the zero-curvature parabolic lines indicated by dashed lines. When the dispersive effects are turned
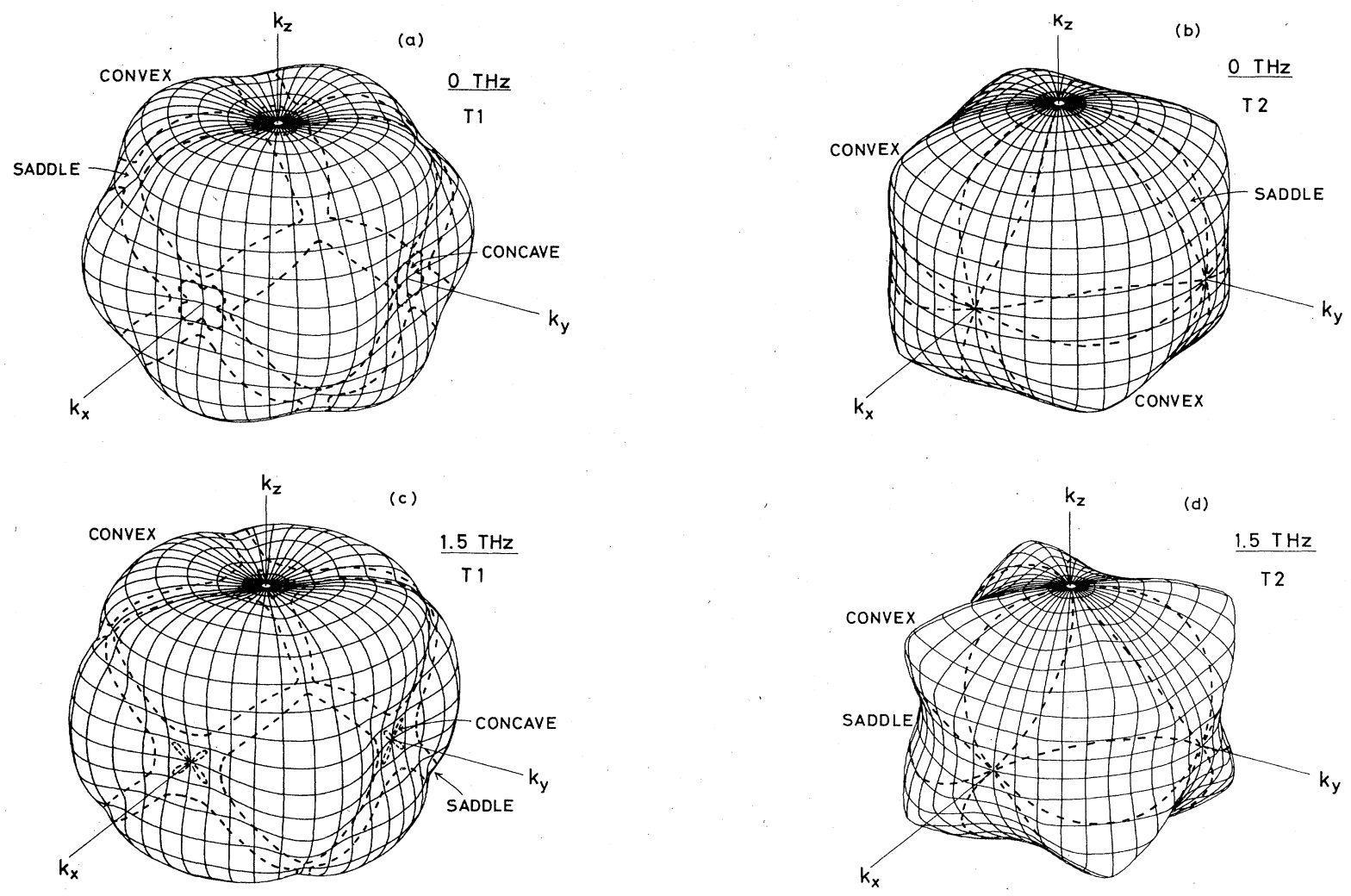

FIG. 2. Constant-frequency surfaces ( $\omega$ surfaces) of TA phonons in GaAs. Dashed lines indicate zero Gaussian curvature and they separate convex, saddle, and concave regions. (a) $\mathrm{T} 1$ mode, $0 \mathrm{THz}$; (b) T2 mode, $0 \mathrm{THz}$; (c) T1 mode, $1.5 \mathrm{THz}$; and (d) T2 mode, $1.5 \mathrm{THz}$. 
on, $\omega$ surfaces are noticeably deformed. Similar to the case in Ge (Ref. 15) the narrowing (spreading) of the saddle regions can be seen on the T1 (T2) surface at the higher frequency. These changes should lead to the significant variations of the phonon-focusing patterns at near-zone-boundary frequencies.

Here, we remark on one of the qualitative differences of the shape of the $\omega$ surfaces for GaAs from those for $\mathrm{Ge}$ being shown in an earlier work. ${ }^{15}$ At $0 \mathrm{THz}$ the $\mathrm{T} 1$ surface of $\mathrm{GaAs}$ possesses a continuous concave area extended around the $\langle 100\rangle$ axis [Fig. 2(a)]. For Ge the corresponding concave region forms a four-leafed clover extended about the $\langle 100\rangle$ axis, which contracts with increasing frequency and vanishes at a frequency about 1.2 $\mathrm{THz}$ (see also Fig. 5). In the continuum approximation the characteristics of the shape of the $\omega$ surfaces for cubic crystals have been examined extensively by Every. ${ }^{16} \mathrm{He}$ has found that the topologies of the $\omega$ surface are classified into several sets by the relative magnitude of elasticconstant ratios $x=C_{11} / C_{44}$ and $y=C_{12} / C_{44}$. On a plot of $x$ versus $y, \mathrm{GaAs}$ is located slightly below the line determined by the equation

$$
(x-1)(x+y)+(x-y-2)(y+1)^{2}=0,
$$

whereas Ge is located slightly above the same line. This is the reason for the above-mentioned difference of the shapes of the T1 surfaces in the vicinity of the fourfold axis at $0 \mathrm{THz}$. However, as the frequency increases, the
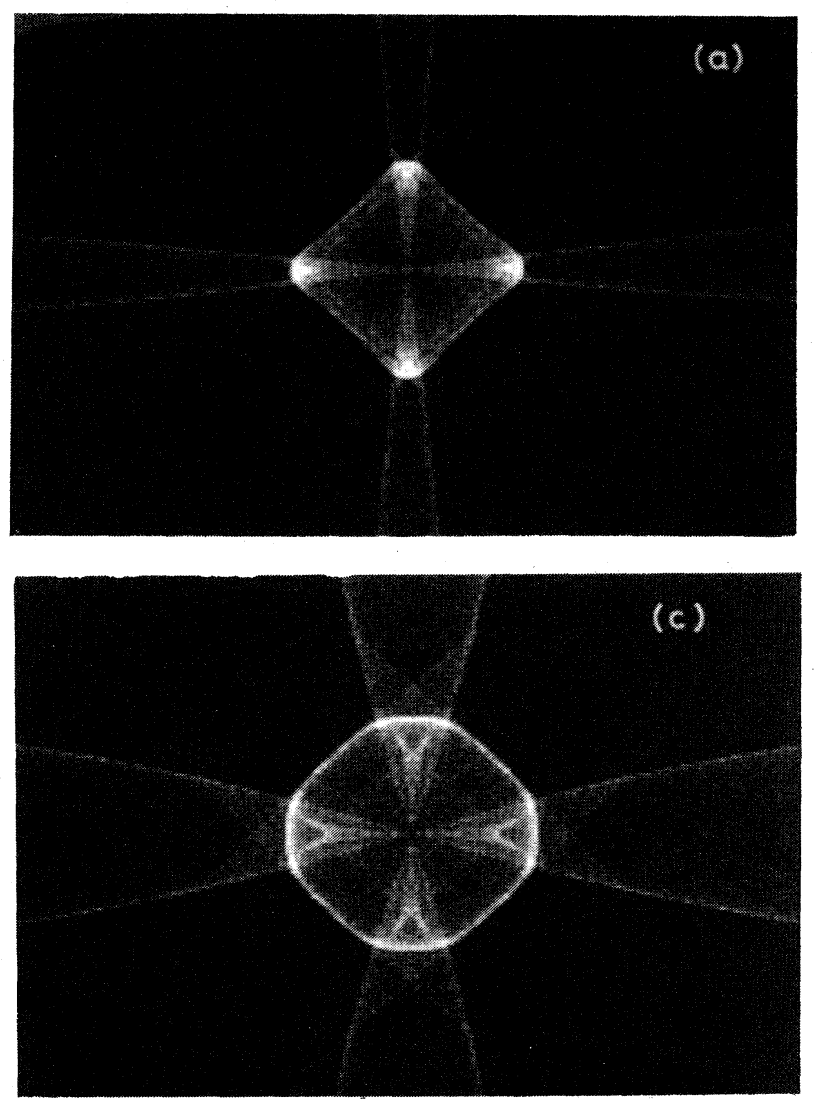

FIG. 3. Simulated ballistic-phonon focusing patterns projected onto the (001) plane of GaAs extending $\pm 39^{\circ}$ horizontally and $\pm 29^{\circ}$ vertically in propagation angle. (a) $0 \mathrm{THz}$, (b) $0.7 \mathrm{THz}$, (c) $1.1 \mathrm{THz}$, and (d) $1.5 \mathrm{THz}$.

above continuous concave region of the $T 1$ surface in $\mathrm{GaAs}$ is deformed also into the four-leafed-clover form, which survives even at near-zero-boundary frequencies, although it becomes more slender at high frequencies. All of these distortions of the $\omega$ surfaces due to the phonon dispersion should be directly reflected in the spatial patterns of the ballistic-phonon flux which will be shown in the next section.

\section{SPATIAL DISTRIBUTION OF PHONONS AND CAUSTICS}

For the comparison with experimental phonon images, it is useful to make a simulation of the phonon flux. A large number of TA-phonon wave vectors (of about $3 \times 10^{5}$ ) are randomly generated in an isotropic distribution and their corresponding group-velocity vectors are computed and projected onto the (001) surface of GaAs. Figures $3(\mathrm{a})-3(\mathrm{~d})$ are the TA-phonon images calculated for $0,0.7,1.1$, and $1.5 \mathrm{THz}$ frequencies. The characteristics of the focusing patterns in GaAs are the presence of heavy accumulation regions of the $\mathrm{T} 1$ phonons around the fourfold $\langle 100\rangle$ axis and of the T2 phonons spreading about the $\{100\}$ plane. In addition, the structures extending from the $\langle 100\rangle$ to $\langle 111\rangle$ axes due to the $\mathrm{T} 1$ phonons do exist. Comparing these structures in the images of the selected frequencies, the dispersive effects on the focusing pattern are really discernible in the image of $0.7 \mathrm{THz}$ and
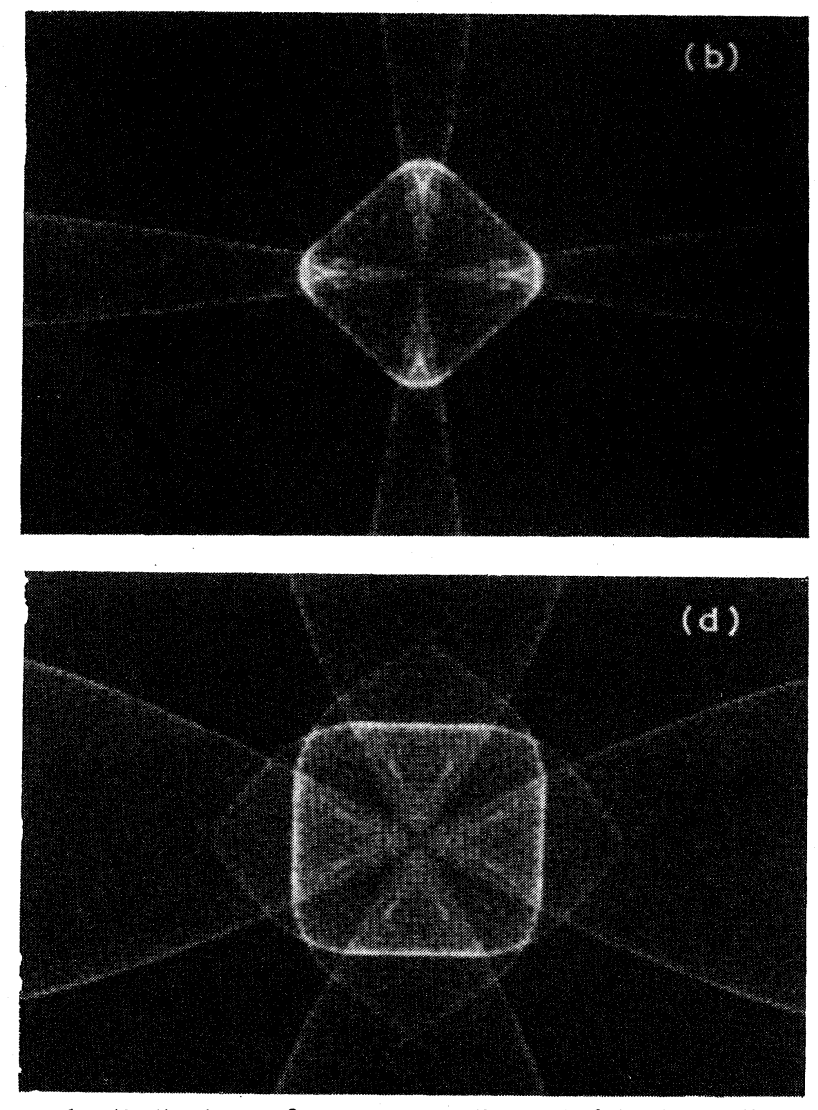
they become significant at frequencies higher than $1 \mathrm{THz}$. More specifically, as the frequency increases the typical square structure of the $\mathrm{T} 1$ phonons being present at 0 $\mathrm{THz}$ becomes gradually round (see especially the image of 1.1 THz) and four pairs of the T2-phonon-focusing region become rapidly wider. Incidentally, we note that the accumulation of the phonons tends to be rather gentle at high frequencies as the focusing regions develop in the real space.

To aid in analyzing further the structures of the focusing patters shown in Fig. 3, it is helpful to identify the directions along which the enhancement factor ${ }^{17}$ of the phonon flux diverges. Such directions originate from the parabolic points on the $\omega$ surfaces and are distributed on the continuous lines or surfaces called caustics in the real space. ${ }^{18}$ The caustics distinctively separate the focusing region from the defocusing region of the phonons. In Figs. 4(a)-4(d) the caustics of the TA phonons in the (001) plane of GaAs are illustrated. The coincidence of the calculated caustic directions with the boundaries of the focusing regions is remarkable. Hence, by measuring the locations of the caustics we can identify, in principle, the maximum frequency of the ballistic phonons detected in the experimental phonon images. In Fig. 5 the theoretical frequency dependences of three loci of the caustics (denoted by polar angles $\theta_{\mathrm{T} 1}, \theta_{\mathrm{T} 1}^{\prime}$, and $\theta_{\mathrm{T} 2}$ ) in the (110) plane of GaAs together with those of Ge (see also Sec. V below) have been plotted. Generally, for the T1 mode, there exist four intersections of the caustics with this plane, i.e., two near the [001] axis and other two near the [111] axis, whereas the T2 mode has only one intersection in the vicinity of the [110] axis (cf. Fig. 4). For the T1 mode the loci of the former two are displayed. The mea-

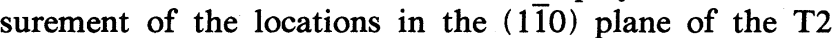
caustic $\left(\theta_{\mathrm{T} 2}\right)$ and the T1 caustic $\left(\theta_{\mathrm{T} 1}\right)$ situated outside, should be best suited for determining the maximum value of the ballistic-phonon frequency. This is because in these directions the phonon intensity suddenly jumps an order of magnitude, ${ }^{19}$ and in addition, their locations are quite sensitive to the phonon frequency. For instance, at $0 \mathrm{THz}$ $\theta_{\mathrm{T} 2}=3.7^{\circ}$ and $\theta_{\mathrm{T} 1}=9.3^{\circ}$ but at $1.5 \mathrm{THz}$ they become $\theta_{\mathrm{T} 2}=16.1^{\circ}$ and $\theta_{\mathrm{T} 1}=17.8^{\circ}$ where $\theta_{\mathrm{T} 1}$ and $\theta_{\mathrm{T} 2}$ are measured from the [001] and [110] directions, respectively.

Now we shall compare our results with the characteris-
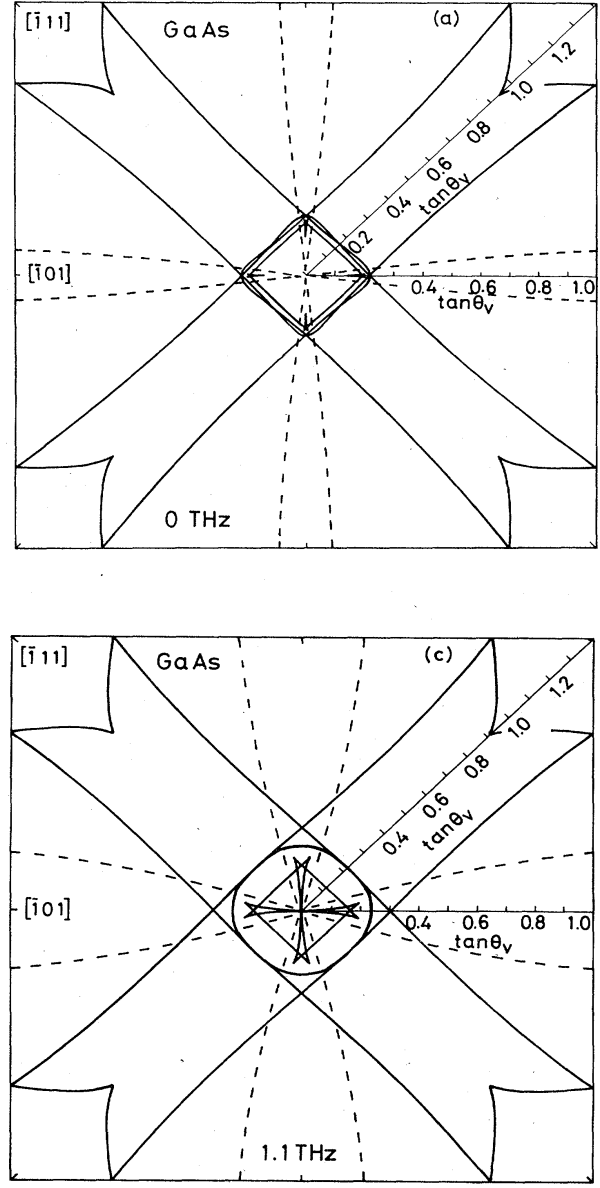
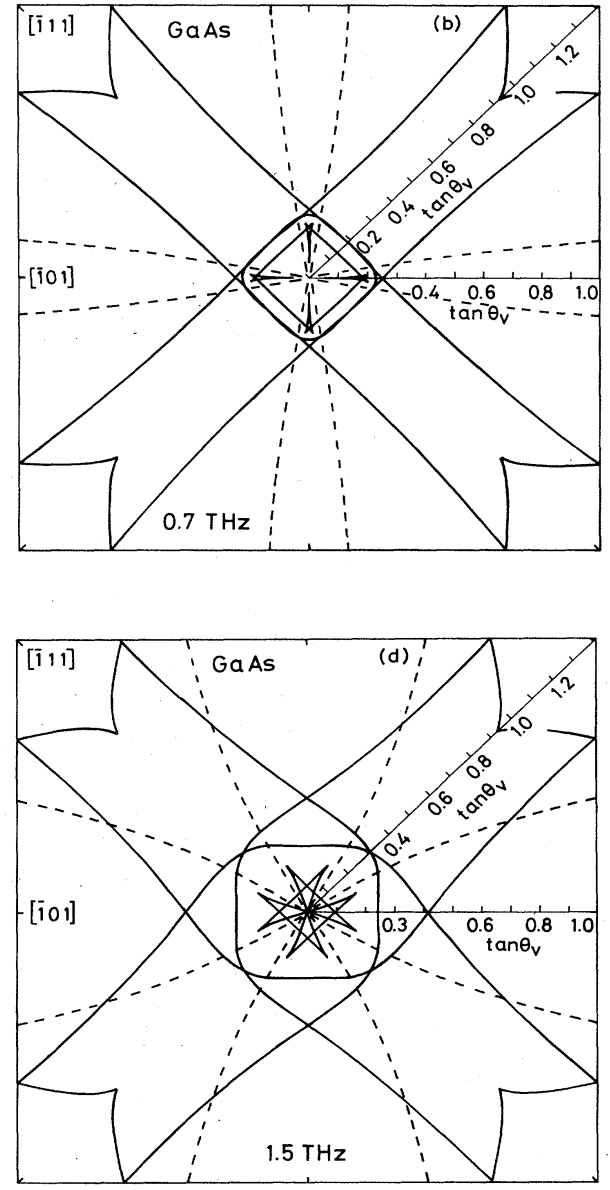

FIG. 4. Theoretical maps of the caustics of GaAs in the (001) plane centered on the [001] direction for both the T1 phonons (solid lines) and the T2 phonons (dashed lines). Frequencies are (a) $0 \mathrm{THz}$, (b) $0.7 \mathrm{THz}$, (c) $1.1 \mathrm{THz}$, and (d) $1.5 \mathrm{THz}$. $\theta_{v}$ indicates the polar angle of group-velocity vector. 


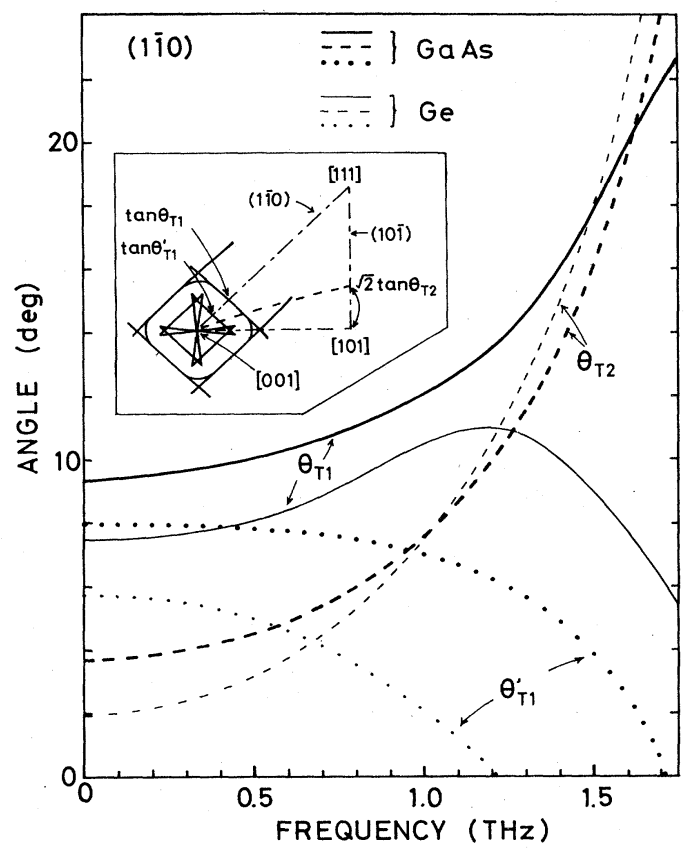

FIG. 5. Frequency dependence of the loci of the caustics in the (110) plane of GaAs (thick lines) and of Ge (thin lines). $\theta_{\mathrm{T} 1}$ and $\theta_{\mathrm{T} 1}^{\prime}$ indicate, respectively, the polar angles for directions of the outside and inside caustics of the $\mathrm{T} 1$ phonons enclosing the [001] axis. $\theta_{\mathrm{T} 2}$ stands for the polar angle for the direction of the $\mathrm{T} 2$ caustic. $\theta_{\mathrm{T} 1}$ and $\theta_{\mathrm{T} 1}^{\prime}$ are measured from the [001] axis and $\theta_{\mathrm{T} 2}$ is measured from the [110] axis.

tic features of the phonon images obtained by Wolfe and Northrop. ${ }^{4}$ They have obtained two phonon images for the 1.9- and $0.25-\mathrm{mm}$-thick samples. The high-frequency phonons in GaAs suffer severely from the scattering by isotopic atoms and their theoretical mean-free paths become shorter than $1 \mathrm{~mm}$ at frequencies higher than 1 THz. ${ }^{5}$ Accordingly, the dispersive shift of the ballisticphonon focusing is naively expected to be observable in samples of submillimeter in thickness.

Comparing the experimental shapes of the focusing patterns with the calculated locations of the caustics, we find that the image for the $1.9-\mathrm{mm}$-thick sample detects ballistic phonons of frequencies up to about $0.7 \mathrm{THz}$. This result is indeed consistent with the mean-free path of 1.5 $\mathrm{mm}$ of $0.7-\mathrm{THz}$ phonons limited by the isotope scattering. ${ }^{5}$ The image for the $0.25-\mathrm{mm}$-thick sample is more obscure as compared to the image of the former sample, making it rather hard to identify the maximum frequency of the ballistic phonons involved. However, from the nearly round shape of the observed focusing patterns of the T1 phonons around the fourfold axis we can conclude that the phonons of frequency up to $1.1 \mathrm{THz}$ travel ballistically over the sample length. This is also in agreement with the predicted mean-free path of about $0.3 \mathrm{~mm}$ for the 1.0-THz TA phonons in GaAs. ${ }^{5}$

\section{COMPARISON WITH DISPERSIVE EFFECTS ON THE FOCUSING IN Ge}

We shall briefly compare the results obtained above for GaAs with the results for Ge. ${ }^{15}$ We may expect that the similar dispersive effects on the TA-phonon focusing should be present in both GaAs and Ge because the dispersion relations for the acoustic branches of these crystals are nearly identical as remarked in Sec. I. To convince ourselves of this expectation, we have plotted in Figs. 6(a)-6(c) the caustic patterns of the TA phonons
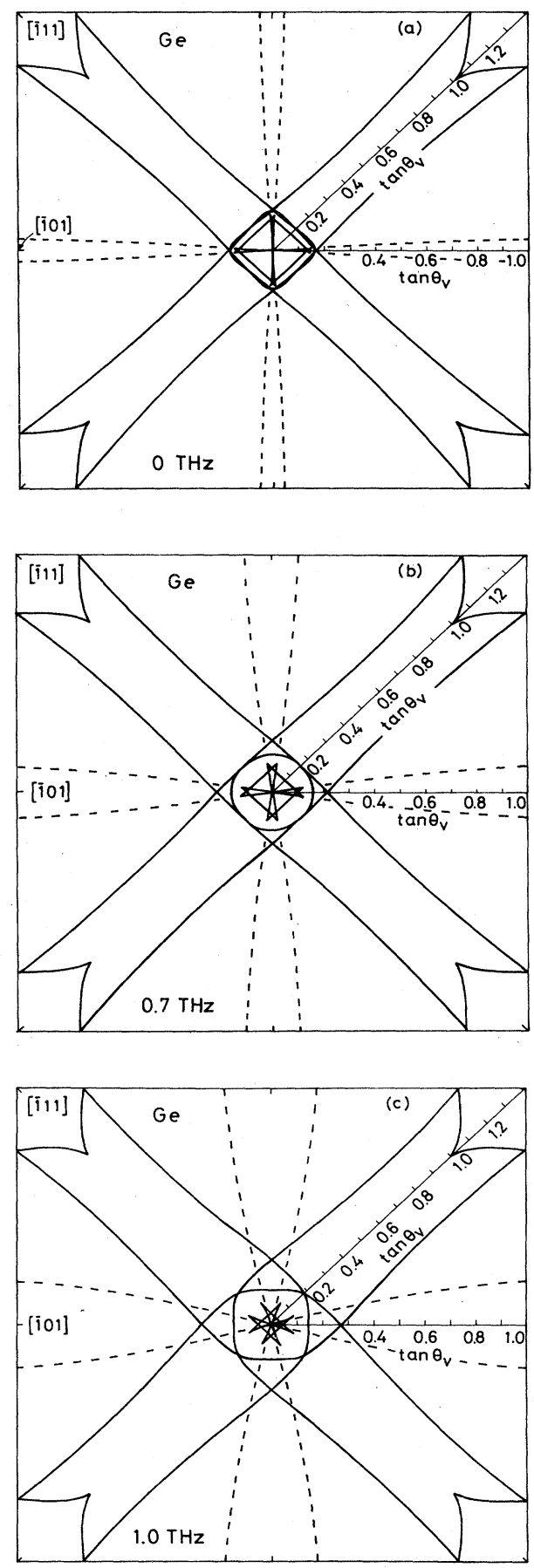

FIG. 6. Theoretical maps of the caustics of Ge in the (001) plane centered on the [001] direction for both the T1 phonons (solid lines) and the $\mathrm{T} 2$ phonons (dashed lines). Frequencies are (a) $0 \mathrm{THz}$, (b) $0.7 \mathrm{THz}$, and (c) $1.0 \mathrm{THz}$. 
projected also on the (001) crystal plane of Ge for $0,0.7$, and $1.0 \mathrm{THz}$, respectively. At $0 \mathrm{THz}$, characteristic square shapes surrounding the fourfold axis are constructed by the caustics of the T1 phonons. Equivalently, this is also seen in GaAs in the low-frequency limit [Fig. 4(a)], although their locations are slightly shifted from each other due to the different magnitudes of the elastic anisotropy. ${ }^{20}$ Surprisingly, however, for Ge these structures of the $\mathrm{T} 1$ phonons are deformed by dispersion and make a circle at a frequency as low as $0.7 \mathrm{THz}$ [Fig. 6(b)]. For $\mathrm{GaAs}$ such a round shape of the caustics is yielded at a much higher frequency about $1.1 \mathrm{THz}$ [Fig. 4(c)]. Moreover, the predicted shape for the $\mathrm{T} 1$ caustics in GaAs at 1.5 THz is very similar to that in Ge at $1.0 \mathrm{THz}$. These results imply that the distinctive features of the dispersive shifts of the T1-phonon-focusing patterns can adequately be observed at $0.7 \mathrm{THz}$ in Ge but they become detectable at a much higher frequency in GaAs. We also find from Fig. 5 that the shift of the T2-phonon caustics in Ge depends on the frequency more abruptly than that in GaAs.

In these respects GaAs may be regarded as less dispersive than $\mathrm{Ge}$ as far as the focusing properties of the TA phonons are concerned. As a result, our model suggests that the distortion of the ballistic-focusing patterns in GaAs is slightly difficult to observe experimentally, as compared to $\mathrm{Ge}$, because the phonons are subject to scattering, very efficiently, at high frequencies.

\section{CONCLUDING REMARKS}

In this paper the dispersive effects on the TA-phonon focusing in GaAs have been investigated on the basis of an extended rigid-ion model. Our analysis implies that the dispersive effects on the directional properties of the phonons in GaAs are less pronounced than in Ge despite the apparent coincidence of the dispersion curves of the TA branches in these crystals. Calculated results exhibit, however, good agreement with the dispersive shifts of the focusing patterns in GaAs obtained by Wolfe and Northrop. ${ }^{4}$ Our results also make it possible to analyze quantitatively the maximum frequency of the phonons which constitute the ballistic component of the experimental images. In the experiment of Wolfe and Northrop the $\mathrm{Al}$ bolometer which is sensitive to the phonons of all frequency range has been used as a detector. ${ }^{4}$ For more precise understanding of the dispersive effects on the phonon focusing a detector such as the $\mathrm{Pb}$-oxide- $\mathrm{Pb}$ tunneling junction which may selectively respond to the nearzone-boundary phonons should be employed. ${ }^{21}$

In this work only spatial distribution of ballistically propagating dispersive phonons is studied. To resolve further the substantial features of the experiment of Ulbrich et al. ${ }^{2}$ the analysis of temporal behaviors of the phonon flux (i.e., line shapes) should be performed by incorporating more rigorous scattering effects (which include anisotropy and dispersion) than those discussed in the literature.

\section{ACKNOWLEDGMENTS}

The authors would like to thank Professor T. Sakuma for a critical reading of the manuscript. This work is partially supported from a Grant-in-Aid for Scientific Research from the Ministry of Education, Science and Culture, Japan.
${ }^{1}$ See, for example, Phonon Scattering in Condensed Matter, edited by $\mathrm{W}$. Eisenmenger, K. Lassmann, and S. Dottinger (Springer, Berlin, 1984).

${ }^{2}$ R. G. Ulbrich, V. Narayanamurti, and M. A. Chin, Phys. Rev. Lett. 45, 1432 (1980).

${ }^{3}$ N. M. Guseinov and Y. B. Levinson, Solid State Commun. 45, 371 (1983); Zh. Eksp. Teor. Fiz. 85, 779 (1983) [Sov. Phys.JETP 48, 452 (1983)].

4J. P. Wolfe and G. A. Northrop, in Phonon Scattering in Condensed Matter, Ref. 1, p. 100.

${ }^{5}$ S. Tamura, Phys. Rev. B 30, 849 (1984).

${ }^{6}$ G. Dolling and J. L. T. Waugh, in Lattice Dynamics, edited by R. F. Wallis (Pergamon, London, 1965), p. 19.

${ }^{7}$ G. Nilsson and G. Nelin, Phys. Rev. B 3, 364 (1971).

${ }^{8}$ M. Lax, Phys. Rev. Lett. 1, 133 (1958).

${ }^{9}$ W. Cochran, Phys. Rev. Lett. 2, 495 (1959).

${ }^{10}$ C. W. Garland and K. C. Park, J. Appl. Phys. 33, 759 (1962).
${ }^{11}$ G. Arlt and P. Quadflieg, Phys. Status Solidi 25, 323 (1968).

${ }^{12}$ K. S. Champlin and G. H. Glover, J. Appl. Lett. 12, 231 (1968).

${ }^{13} \mathrm{~S}$. Tamura (unpublished).

${ }^{14}$ B. Taylor, H. J. Maris, and C. Elbaum, Phys. Rev. B 3, 1462 (1971).

${ }^{15}$ S. Tamura, Phys. Rev. B 28, 897 (1983).

${ }^{16}$ A. G. Every, Phys. Rev. B 24, 3456 (1981).

${ }^{17}$ H. J. Maris, J. Acoust. Soc. Am. 50, 812 (1971).

${ }^{18}$ P. Taborek and D. L. Goodstein, Solid State Commun. 33, 1191 (1980).

19J. C. Hensel and R. C. Dynes, Phys. Rev. Lett. 43, 1033 (1979).

${ }^{20}$ Anisotropy factor defined by $2 C_{44} /\left(C_{11}-C_{12}\right)$ is 1.83 for $\mathrm{GaAs}$ and 1.67 for $\mathrm{Ge}$.

${ }^{21}$ W. Dietsche, G. A. Northrop, and J. P. Wolfe, Phys. Rev. Lett. 47, 660 (1981). 

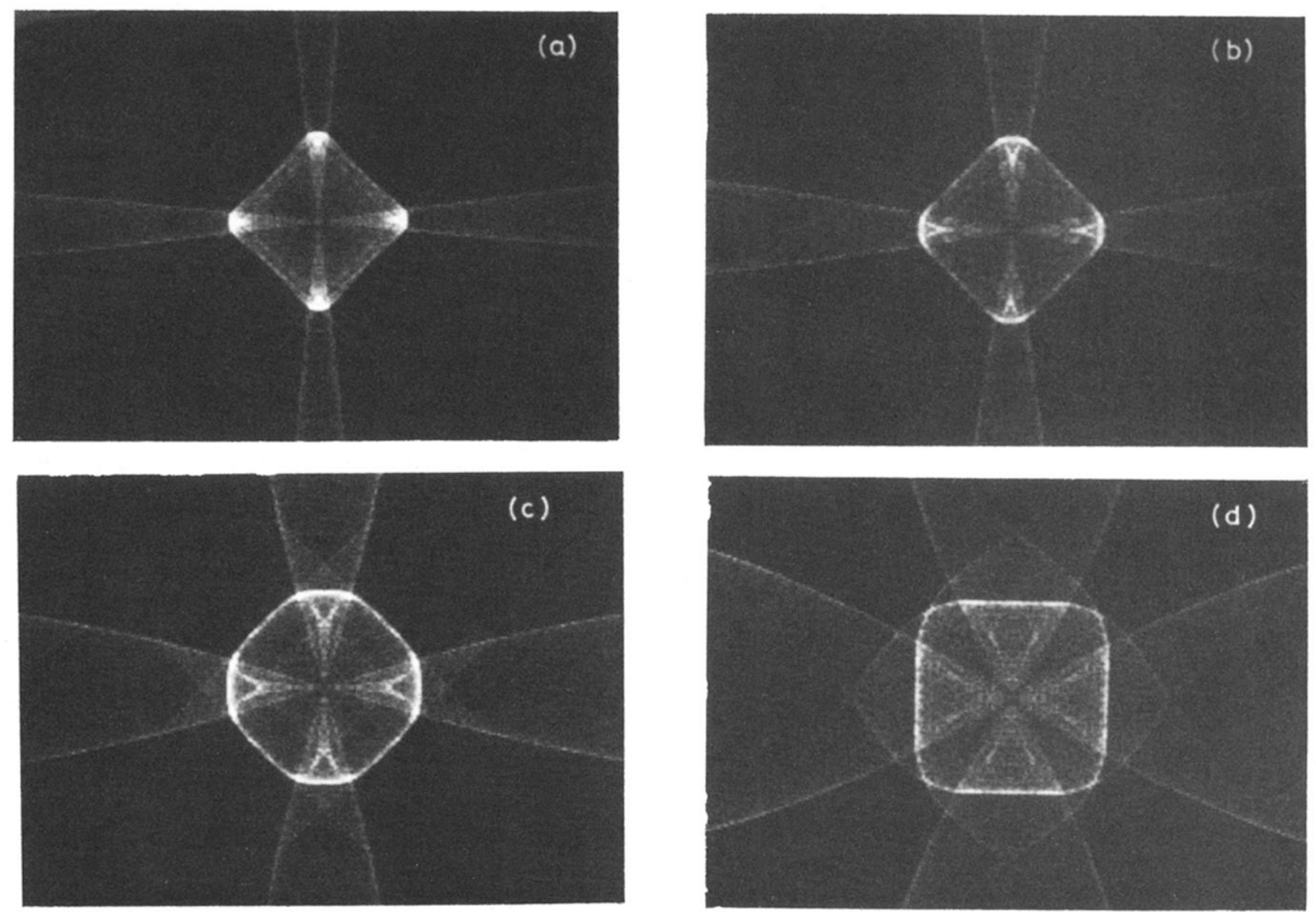

FIG. 3. Simulated ballistic-phonon focusing patterns projected onto the (001) plane of GaAs extending $\pm 39^{\circ}$ horizontally and $\pm 29^{\circ}$ vertically in propagation angle. (a) $0 \mathrm{THz}$, (b) $0.7 \mathrm{THz}$, (c) $1.1 \mathrm{THz}$, and (d) $1.5 \mathrm{THz}$. 